\title{
Review of: "Resistance to a CRISPR-based gene drive at an evolutionarily conserved site is revealed by mimicking genotype fixation"
}

\author{
Vanessa Macias ${ }^{1}$ \\ 1 Pennsylvania State University
}

Potential competing interests: The author(s) declared that no potential competing interests exist.

A review of "Resistance to a CRISPR-based gene drive at an evolutionarily conserved site is revealed by mimicking genotype fixation." by Fuchs et al. 2021, BioRxiv

Vanessa Macias, September 2021

\section{Summary:}

Cas9-based gene-drives that target an essential mosquito gene are predicted to spread through and suppress a vector population and so are being designed to control mosquitoes and mosquito-borne pathogens. Because mutations at the Cas9 target site on the mosquito genome can impede the efficacy of the drive strategy, a highly conserved site is thought to be optimal, so that natural variation in a population at that site is minimal. However, it is possible that new mutations to the target site generated by the drive itself through Cas9-mediated cleavage and so resistance can still arise in a population that is homogenous at given target site ("ultra-conserved" sites), especially because, as in a suppression strategy, the fitness load of the drive on the population becomes very high. The authors here present a set of genetic assays that experimentally confirm that even an ultra-conserved site can become resistant to the suppressive drive within generations. Importantly, this strategy can be used in efforts to develop future suppression-type gene drives to test the resilience of a conserved genomic location to drive resistance and so this work constitutes a good approach for characterizing potential target gene. Such efforts in future designs of Cas9-based suppressive gene drives will support a successful implementation of this promising technology for mosquito control.

As a target for a transgenic drive construct, the authors select an essential gene that is not only conserved among wild An. gambiae, but also highly conserved in sequencing data from many Anopheles species. Two transgenic lines, one with a control construct that simply interrupts the target gene and marks the null allele and one that encodes a Cas9 and guide RNA for drive of the construct.

The authors present a fairly thorough characterization of the two lines and using the marked control line along with the gene drive line allowed the authors to tease apart effects of gene knockout from the fitness load of the drive components of the construct and demonstrate a cost from both. The authors conclude 
that the gene drive construct added a fitness cost over the cost of the loss of the essential gene, based both on the failure of the drive construct to persist (much less spread) in a seeded cage population and based on modeling of allele proportions.

Some clarification on the conclusion that AGAP029113 as a truly haplosufficient gene is needed. The authors mention that no apparent fitness cost was seen during maintenance of the heterozygotes of the marker-only construct (29113 ${ }^{\mathrm{hdrGFP} /+}$ ) and in figure 2, include measurements of egg laying counts and hatching proportions from outcrosses of the heterozygotes to wild-types but only the progeny of a few individuals are included in the graph for eclosion rate to adults (Fig 2E) from a minimum of 15 females (if I'm understanding the methods correctly) along with data collected from the outcross of the gene-drive line (29113 ${ }^{\mathrm{CRISPRh} /+}$ ). If only 3-4 individuals had larvae that resulted in adults out of 15 , this is important data for understanding the viability phenotype of individuals with only one functional copy, especially because the gene is described in homozygous individuals to result in failure to emerge as adults. This question of whether one intact copy of this gene is sufficient for full gene function or not does not undermine the impact of the paper though, especially because the authors lay the groundwork for assessing this phenotype for potential target genes.

The control line also allowed the authors to test for mutations that maintained gene function after Cas 9 targeting using a cross of heterozygotes for the marked null allele (GFP+) to heterozygotes for the drive construct (RFP+). Selection and molecular analysis of GFP-only progeny allowed to authors to identify chromosomes that had been cleaved, but not repaired by HDR insertion of the drive AND that were viable at either larvae or adult stages. Several potential indels were experimentally identified and the authors reasonably suggest that those present in larvae, but not adults, were not fully functional.

A cage assay with intercrossing of the gene-drive line was used, instead of seeding a wild-type population, to replicate a mostly suppressed population resulting in the observation that a functional mutant emerged within only several generations and outcompeted the drive, reversing the suppression of the population. Further that this was one of the functional mutations observed in the non-drive assay is interesting and validates the utility of the resistance assay for identifying potential mutant alleles. While this cage assay was not replicated and the propensity for this to occur was not quantified, the data non-the-less support the conclusion that this rescue mutation can occur even at a highly-conserved site, demonstrating that this site has some functional flexibility. This also supports the authors premise that "totally conserved" does not necessarily mean totally functionally inflexible. It will be interesting to probe what the range of this flexibility is among highly conserved sites and whether there are sites that are totally inflexible.

The results presented here give both an impetus and methods for characterizing genes with potential as suppression drive targets using careful assessment of the target gene function and phenotype and 
emphasize the need to characterize and minimize the fitness load of the drive components of the construct.

A minor correction: on line 592: U6 is a pol III promoter

For clarity, can you explain the purpose of the RFP marker in the construct on Fig 1A? 